\title{
Concentração de benzeno: modelo de monitorização em medicina do trabalho
}

\author{
Benzene concentration: monitoring model in labor medicine \\ Concentración de benceno: modelo de monitoreo en medicina del trabajo \\ Cristiane Figueiredo Reis Maiorquin ${ }^{1 *}$.
}

\begin{abstract}
RESUMO
Objetivo: Avaliar a eficácia do equipamento de proteção individual numa população exposta ao benzeno considerando os níveis de absorção medidos através do ácido trans-trans mucônico. Métodos: Trata-se de ensaio experimental randomizado. Estudou-se o comportamento do ácido trans-trans mucônico (ATTM) em amostras de urina de trabalhadores expostos ao benzeno. Foram selecionados 58 frentistas que trabalhavam na bomba de gasolina em uma cidade do estado de Rondônia. Na primeira coleta nenhum participante utilizou equipamento de proteção individual (EPI). Em seguida houve intervenção. Foram escolhidos aleatoriamente 30 participantes, para utilizar EPI por 2 dias consecutivos. No segundo dia, realizou-se nova coleta ao final da jornada de 12 horas de trabalho de todos participantes. A concentração do ATTM foi determinada pelo método de Cromatografia líquida de alta eficiência. Os dados foram analisados pelo programa SPSS 20.0, utilizando-se a prova de normalidade, a prova t para amostras relacionadas, aceitando como significativo $p<0,05$. O estudo foi aprovado por Comitê de Ética em Pesquisa. Resultados: O trabalho demonstrou diminuição no valor de ATTM nos participantes utilizando EPI, com $p$ significativo $(p=0,006)$. Não houve diferença significativa no grupo que não utilizou EPI $(p=0,869)$. Conclusão: $A$ investigação demonstrou importância dos EPI para saúde pública e ocupacional.
\end{abstract}

Palavras-chave: Benzeno, Equipamento de proteção individual, Exposição ocupacional.

\begin{abstract}
Objective: To evaluate the effectiveness of personal protective equipment in a population exposed to benzene considering absorption levels measured through trans-trans muconic acid. Methods: This is a randomized experimental trial. Trans-trans muconic acid (TTMA) behavior was studied in urine samples of workers exposed to benzene. Fifty-eight gas station attendants who worked at the gas station in a city in the state of Rondônia were selected. In the first collection no participant used personal protective equipment (PPE). Then there was intervention. Thirty participants were randomized to use PPE for 2 consecutive days. On the second day, a new collection took place at the end of the 12-hour working day of all participants. TTMA concentration was determined by high performance liquid chromatography method. The data were analyzed by the SPSS 20.0 software, using the normality test, the $t$ test for related samples, accepting as significant $p<0.05$. The study was approved by the Research Ethics Committee. Results: The study demonstrated a decrease in TTMA value in participants using PPE, with significant $p(p=0.006)$. There was no significant difference in the group that did not use PPE $(p=0.869)$. Conclusion: Research has shown the importance of PPE for public and occupational health.
\end{abstract}

Keywords: Benzene, Personal protective equipment, Occupational exposure.

\section{RESUMEN}

Objetivo: evaluar la efectividad del equipo de protección personal en una población expuesta al benceno considerando los niveles de absorción medidos a través del ácido trans-trans mucónico. Métodos: Este es un ensayo experimental aleatorio. El comportamiento del ácido trans-trans mucônico (ATTM) se estudió en muestras de orina de trabajadores expuestos al benceno. Se seleccionaron 58 asistentes de estaciones de gasolinerias que trabajaban en la bomba de gasolina en una ciudad en el estado de Rondônia. En la primera colección, ningún participante usó equipo de protección personal (EPP). Luego hubo intervención. Treinta participantes fueron asignados al azar para usar EPP durante 2 días consecutivos. El segundo día, se realizó

${ }^{1}$ Instituto Universitário Italiano de Rosário (IUNIR), Rosário-Santa, Fé-ARG. *E-mail: crismaiorquin@bol.com 
una nueva recolección al final del día laboral de 12 horas de todos los participantes. La concentración de ATTM se determinó mediante un método de cromatografía líquida de alto rendimiento. Los datos fueron analizados por el software SPSS 20.0, utilizando la prueba de normalidad, la prueba $t$ para muestras relacionadas, aceptando como significativo $p<0.05$. El estudio fue aprobado por el Comité de Ética en Investigación. Resultados: El estudio demostró una disminución en el valor de ATTM en los participantes que usan PPE, con una $p$ significativa $(p=0.006)$. No hubo diferencias significativas en el grupo que no utilizó EPP $(p=0,869)$. Conclusión: La investigación ha demostrado la importancia del EPP para la salud pública y ocupacional.

Palabras clave: Benceno, Equipo de protección personal, Exposición ocupacional.

\section{INTRODUÇÃO}

Há mais de 100 anos são conhecidos os efeitos deletérios do benzeno na saúde do homem. Desde 1897 encontramos informes na literatura científica sobre os efeitos da exposição repetida ao benzeno em trabalhadores (MENDES R, 2013).

Hunter FT (1939) fez descrições clínicas detalhadas, que mostraram uma alta incidência de achados multiformes hematológicos que afetavam a série vermelha, série branca e plaquetas. A atenção se concentrava na anemia aplástica ou na insuficiência da médula óssea, que representava em muitos casos, uma fase terminal e irreversível. Os informes mais dramáticos fizeram menção de exposição a níveis de benzeno que iam desde 75 partes por milhão (ppm) a aproximadamente 1300 ppm.

O benzeno é um hidrocarboneto aromático, de fórmula química $\mathrm{C} 6 \mathrm{H} 6$, líquido, incolor, volátil, com um ponto de ebulição de $80,1 \circ \mathrm{C}$, inflamável, com uma alta solubilidade em lípidos e praticamente insolúvel em água. É largamente utilizado como matéria-prima para diversos produtos, como detergentes, gasolina e plásticos (COTTICA D e GRIGNANI E, 2013). Sua utilização industrial no Brasil vem diminuindo progressivamente em virtude da proibição de seu uso como solvente industrial, mas apesar disto, este hidrocarboneto ainda representa risco ocupacional para milhões de pessoas (BRASIL, 2006).

A absorção do benzeno ocorre pelas vias cutânea e pulmonar. Cerca de 50 a $90 \%$ do benzeno inalado será absorvido. Sua concentração no sangue alcança um pico máximo em alguns minutos, mas decai com a rápida liberação do composto para tecidos ricos em lipídios. Cerca de $12 \%$ do benzeno é eliminado pelos pulmões e apenas 0,1 a 0,2\% na urina. A maior parte da fração absorvida (84 a 89\%) é biotransformada no fígado e em menor proporção na medula óssea dos indivíduos expostos (MACEDO RB, 2008).

$\mathrm{Na}$ busca de um indicador biológico efetivo para estudo da exposição ao benzeno, em substituição ao fenol urinário, o ácido trans, trans-mucônico (ATTM) tem sido apresentado como excelente alternativa (COSTA MFB, 2001).

A correlação entre ATTM e benzeno é detectada em níveis ambientais do solvente inferiores a 1,0 ppm, fato que o viabiliza como biomarcador no Brasil, considerando que a concentração de benzeno permitida no ar ocupacional (Valor de Referência Tecnológica- VRT) varia de 1,0 ppm para indústrias químicas e 2,5 ppm para indústrias de siderurgia, depois da celebração do Acordo Nacional sobre o Benzeno criado pela Portaria $\mathrm{N}$ ㅇ 14, 1995 Ministério do Trabalho e Emprego (BRASIL, 2006).

De fato, os estudos epidemiológicos de avaliação de riscos, realizada pela Ocupacional Safety and Health Administration (OSHA), estimam que o nível de exposição de 1 ppm em toda a vida laboral, produz un excesso de 95 mortes por leucemia em cada 1000 trabalhadores que se encontravam expostos ao benzeno (COSTA DF, 2009).

Existem inúmeras doenças causadas pelo benzeno em decorrência do caráter mielotóxico, leucemogênico e cancerígeno do agente químico, mesmo em concentrações consideradas baixas (BRASIL, 2006). Entre elas, leucemia mieloide aguda, leucemia mieloide crônica, leucemia linfocítica crônica e linfoma de Hodking. Segundo a International Agency for Research on Cancer (IARC), o benzeno foi classificado no Grupo A1 das substâncias químicas de exposição ocupacional cancerígenas para os seres humanos (IARC, 1989).

Segundo a diretoria da Federação Nacional dos Empregados em Postos de Serviços de Combustíveis e Derivados de Petróleo (FENEPOSPETRO), eleita para o mandato de 2016-2020, existem aproximadamente 
600 mil trabalhadores de postos de combustíveis em todo país. Uma pesquisa estimou em 2016 que 770.212 trabalhadores sofreram exposição ao benzeno, com uma prevalência de $0.9 \%$ por grupo ocupacional (CORRÊA MJM e SANTANA VS, 2016). No Brasil a exposição ocupacional aos solventes orgânicos é um problema de saúde pública, sem notificação e negligenciados pelos administradores de postos de combustíveis (CERQUEIRA GS et al., 2010).

Os frentistas estão expostos a diversos tipos de combustíveis (gasolina, etanol, diesel, biodiesel, gás natural veicular) e a poluição ambiental, podendo executar seu processo de trabalho em vários setores como troca de óleo e outros lubrificantes, descarregamento de caminhões-tanque, abastecimento de veículos, lavagem de carros ou até mesmo atendimento administrativo. Em sua grande maioria estes trabalhadores não utilizam EPI individual ou coletivo durante o trabalho (CORREA MJ e LARENTIS AL, 2017). Vale ressaltar que a toxicidade do benzeno é maximizada através da exposição simultânea com outros hidrocarbonetos aromáticos como tolueno e xileno (MORO AM, et al., 2019).

A Legislação Internacional, a Organização Mundial de Saúde (OMS), reconhece que não existe um limite seguro de exposicão que se possa recomendar (OGA S, et al., 2008). O benzeno apresenta toxicidade mesmo em doses inferiores a 1 ppm, e o Programa das Nações Unidas de Segurança Química o considera a quinta substância de maior risco químico (MORIYAMA IN, et al., 2017). A exposição ao benzeno no ambiente ocupacional está diretamente relacionada à falta de compromisso e obediência das normas de segurança de trabalho, informações deficientes, supervisionamento inadequado, falta de treinamento, processos de trabalhos ultrapassados e acima de tudo ausência de equipamentos de proteção (PEZZAGNO G, 1995).

A aquisição do EPI adequado ao risco, em perfeito estado de conservação e funcionamento, é obrigação da empresa, sem custo algum para o empregado, atendendo situações de emergência e protegendo contra doenças ocupacionais e riscos de acidentes de trabalho (HOEPPNER MG, 2008). O presente estudo tem como objetivo avaliar a eficácia do equipamento de proteção individual numa população exposta ao benzeno, considerando os níveis de absorção medidos através do ATTM urinário em uma cidade do estado de Rondônia no ano de 2014.

\section{MÉTODOS}

Trata-se de um ensaio experimental randomizado. Foi realizado o estudo em uma população de frentistas em uma cidade do estado de Rondônia, Brasil. Foram convidados a participar da pesquisa trabalhadores de 7 postos de gasolina, sendo obedecidos os seguintes critérios de inclusão: frentistas de ambos os gêneros, entre 18 e 50 anos, que aceitassem participar da pesquisa, assinassem o termo de consentimento livre e esclarecido e que trabalhavam há mais de seis meses no estabelecimento. E os critérios de exclusão: frentistas que já tiveram algum diagnóstico de câncer, gestantes, aqueles que tinham menos de 6 meses de trabalho no estabelecimento, ou que trabalhavam em setor administrativo e aqueles que não aceitassem assinar o termo de consentimento livre e esclarecido.

A população estudada foi de aproximadamente 504 pessoas segundo o Sindicato dos Trabalhadores de Comércio de Minérios e derivados do Petróleo no estado de Rondônia (STCMDP/RO), distribuídos em 56 postos de gasolina na cidade em março de 2014. Nossa amostra foi de 58 frentistas, calculada pelo programa Análise Epidemiológica de Dados Tabulados (EPIDAT), com um nível de confiança de $95 \%$ e com uma precisão de 5, se calculou o tamanho mínimo da amostra para realizar uma estimativa de proporções. A variável dependente da pesquisa foram os níveis de ATTM urinário encontrados nos resultados de exames dos participantes.

A variável independente foi o uso do equipamento de proteção individual (EPI). Foram analisadas também algumas variáveis de controle: sexo, idade, conhecimento sobre a utilização do EPI para a segurança e tempo que trabalhavam no estabelecimento. Também se aplicou um questionário para se obter informações acerca das características da população. Depois da entrevista e cumprimento dos critérios de inclusão, se obtiveram os 58 participantes. As amostras de urina foram coletadas em coletores universais de plástico, de $50 \mathrm{ml}$, no término da jornada de trabalho. Os frascos foram imediatamente fechados e mantidos sobre refrigeração $(4 \circ \mathrm{C})$ e enviadas ao laboratório.

A análise química foi determinada por Cromatografia Líquida de Alta Eficiência. O laboratório escolhido (Bio check-up / Hermes Pardini) tem um método padronizado, validado e participa de programa de controle de qualidade interlaboratorial e intralaboratorial para garantia de confiabilidade analítica de seus resultados. 
As análises estatísticas foram desenvolvidas pelo Programa Statistical Package for the Social Sciences (SPSS) 20.0 para Windows, aceitando significativo estatisticamente um valor de $p<0,05$ similar a outros estudos médicos.

Foram oferecidas aos participantes do grupo intervenção, máscaras do tipo Respirador Purificador de ar tipo peça semifacial filtrante para partículas com FBC1 (classe de filtro para gases e vapores da Norma Brasileira NBR 13696:2005 - Equipamentos para Proteção Respiratória - Filtros Químicos e combinados). Garante proteção contra poeira, névoas, fumos e radionuclídeos, contra vapores orgânicos ou gases ácidos em ambientes com concentração inferior a 50 ppm. Este modelo de máscara possui Certificado de Aprovação (CA) pelo Ministério do Trabalho e Emprego (MTE) e é produzida pela empresa 3M do Brasil.

Inicialmente houve uma coleta do material (urina) de todos aos participantes de 7 postos de gasolina $(\mathrm{N}=58)$. Na semana seguinte, houve a intervenção, e foram selecionados aleatoriamente 30 frentistas para utilizarem o EPI por 2 dias consecutivos de trabalho $(\mathrm{N}=30)$. Ao final da jornada de 12 horas de trabalho do $2^{\circ}$ dia consecutivo, procedeu-se a $2^{\text {a }}$ coleta de urina de todos os participantes, tanto os que usaram o EPI, quanto os que não utilizaram.

A presente pesquisa teve aprovação do Comitê de Ética em Pesquisa com o Certificado de Apresentação de Apreciação Ética de ํㅜ 14909813.2.0000.0012 e Parecer de ํㅜ 427.107. Os frentistas que aceitaram participar da pesquisa assinaram um termo de consentimento livre e esclarecido, conforme exigência do Ministério da Saúde, e foi garantido o anonimato, segundo a resolução № 196/1996 do Conselho Nacional de Saúde sobre pesquisa envolvendo seres humanos.

\section{RESULTADOS}

No segundo semestre de 2014, foram avaliados 58 participantes da pesquisa escolhidos aleatoriamente dentre 7 postos de gasolina em uma cidade do estado de Rondônia. Os trabalhadores eram de ambos os sexos, com idades entre 18 e 55 anos de idade, e trabalhavam como frentistas diretamente na bomba de gasolina. Foram excluídos da pesquisa mulheres grávidas, trabalhadores do setor administrativo, aqueles com menos de 6 meses de emprego e aqueles que alguma vez já haviam sido diagnosticados com neoplasia.

O cálculo do tamanho da amostra (58) foi realizado pelo programa Dados Epidemiológicos Tabulados (EPIDAT), versão $3.1 \mathrm{com}$ um tamanho populacional de 504 frentistas, uma proporção esperada de $95 \%$, um nível de confiança de $95.0 \%$. Destes 58 participantes, 19 (32,76\%) eram do sexo feminino e $39(67,24 \%)$ eram do sexo masculino (Gráfico 1).

Gráfico1 - Distribuição de sexo nos trabalhadores que participaram do estudo.

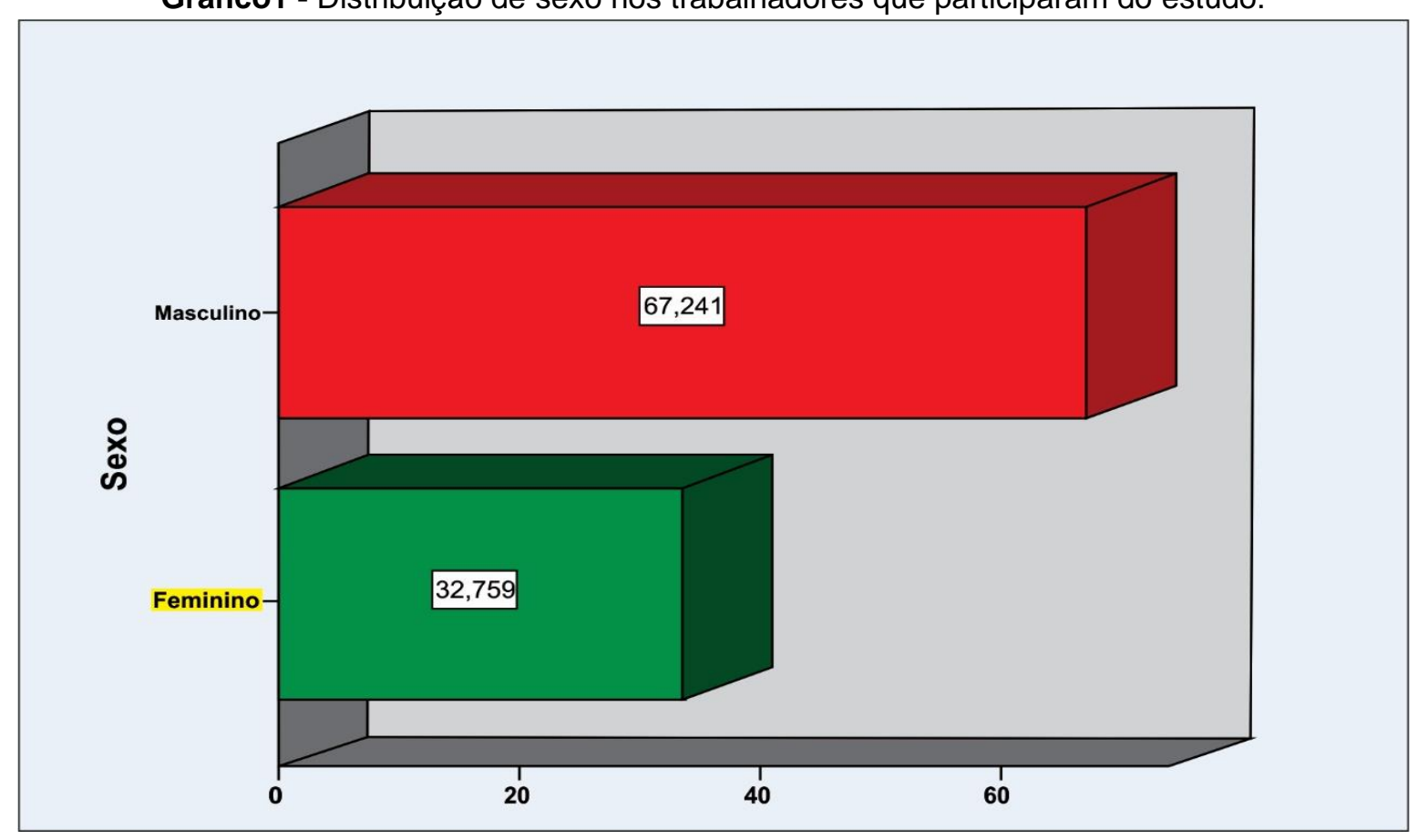

Legenda: sexo X porcentagem. Fonte: Maiorquin CFR, 2015. 
Quanto a distribuição da idade, o grupo que não utilizou o equipamento de proteção individual ( $\mathrm{N}=28)$ correspondeu a média de 28,71 anos, e o grupo que utilizou o equipamento de proteção individual $(\mathrm{N}=30)$ correspondeu a média de 27,76 anos de idade. Em relação ao tempo de emprego, o grupo que não utilizou o equipamento de proteção individual ( $N=30)$, teve em média 28,43 meses de emprego e o grupo que o utilizou $(\mathrm{N}=28)$ teve em média 17,07 meses de emprego. Os participantes da pesquisa também responderam se possuíam algum conhecimento ou não sobre o equipamento de proteção individual que era recomendado para ser utilizado durante o serviço.

A maioria dos participantes $(91,37 \%)$ respondeu que conhecia o mesmo, porém, nenhum dos frentistas que participaram desta pesquisa utilizava o equipamento de proteção individual durante a intervenção. Os gerentes dos postos afirmavam que era disponibilizado aos trabalhadores o equipamento de proteção individual, conforme determinação do Ministério do Trabalho e Emprego (MTE) porém eles não poderiam obrigá-los a utilizar o mesmo. Quanto aos níveis do ATTM urinário, não houveram diferenças significativas no grupo que não utilizou o equipamento de proteção individual entre as duas coletas de ATTM-u, antes e após a intervenção, com um valor de $p=0,869$ (Gráfico 2).

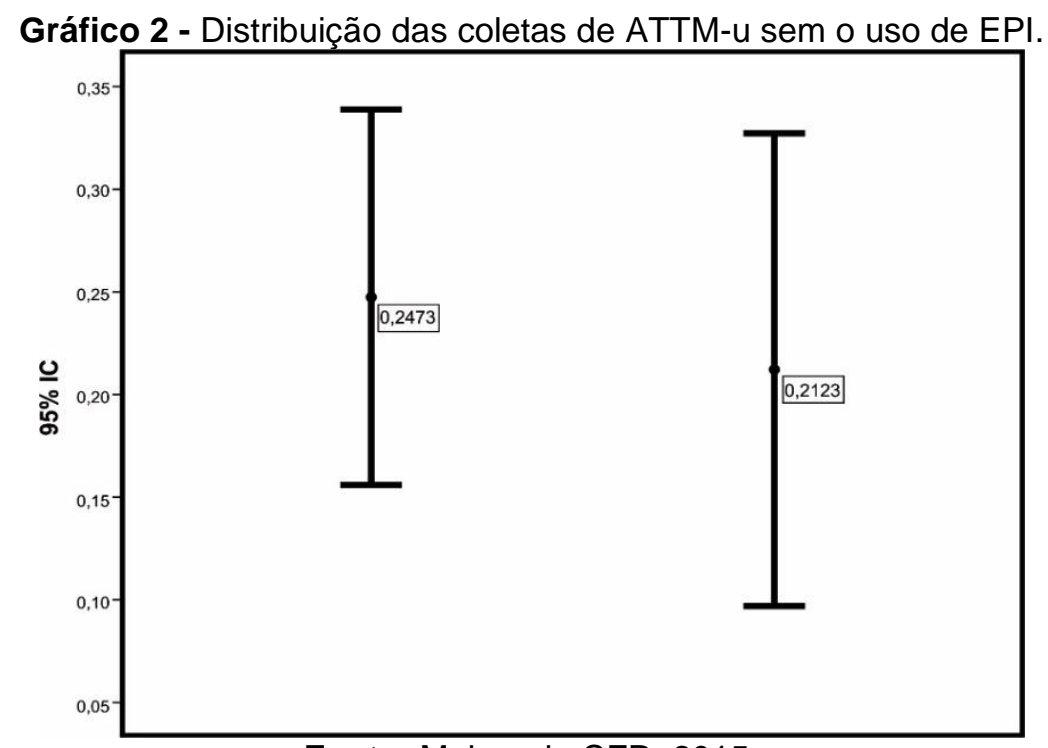

Fonte: Maiorquin CFR, 2015.

Porém, no grupo que utilizou o equipamento de proteção individual, se pode demonstrar que houve uma diferença, uma diminuição estatisticamente significativa no valor do ATTM urinário, comparando-se as duas coletas, antes e após a intervenção com o uso do equipamento de proteção individual, com um valor de $p=$ 0,006 (Gráfico 3).

Gráfico 3 - Distribuição das coletas de ATTM-u com o uso de EPI.

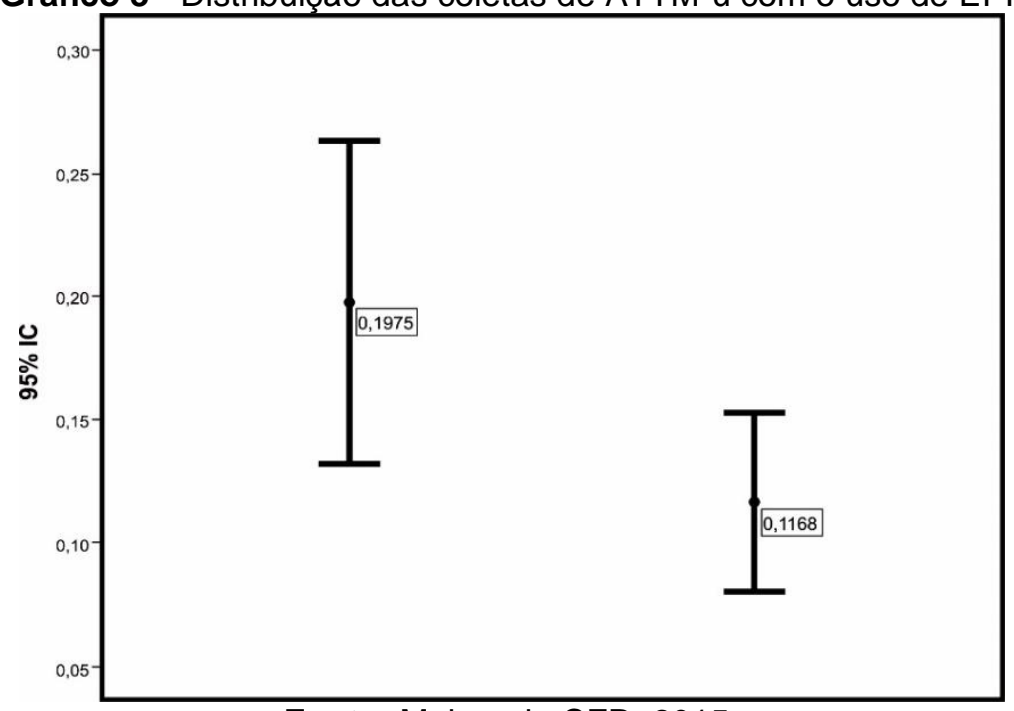

Fonte: Maiorquin CFR, 2015. 
Foi utilizada a prova t para amostras relacionadas com um intervalo de confiança de $95 \%$ para verificar a associação entre os níveis de ATTM urinário em relação ao uso do equipamento de proteção individual aceitando como significativo um valor de $p<0,05$ (Tabela 1).

Tabela 1 - Associação entre os níveis de ATTM-u em relação ao uso de EPI.

\begin{tabular}{|c|c|c|c|c|c|c|c|c|c|}
\hline \multicolumn{10}{|c|}{ Prova de amostras relacionadas } \\
\hline \multirow{3}{*}{\multicolumn{2}{|c|}{ Uso de EPI }} & \multicolumn{5}{|c|}{ Diferenças relacionadas } & \multirow{3}{*}{$\mathbf{t}$} & \multirow{3}{*}{ gl } & \multirow{3}{*}{$\begin{array}{c}\text { Sig } \\
\text { (bilateral) }\end{array}$} \\
\hline & & \multirow[t]{2}{*}{ Média } & \multirow[t]{2}{*}{$\begin{array}{l}\text { Desvio } \\
\text { padrão }\end{array}$} & \multirow[t]{2}{*}{$\begin{array}{l}\text { Erro típ. } \\
\text { da média }\end{array}$} & \multicolumn{2}{|c|}{$\begin{array}{c}\text { 95\% Intervalo de } \\
\text { confiança para a } \\
\text { diferença }\end{array}$} & & & \\
\hline & & & & & Inferior & Superior & & & \\
\hline Sim & $\begin{array}{c}\text { 1a Coleta } \\
\text { ATTM-u (mg/g } \\
\text { de creatinina) - } \\
\text { 2a Coleta } \\
\text { ATTM-u (mg/g } \\
\text { de creatinina) }\end{array}$ & ,08071 &, 14267 & ,02696 & ,02539 & ,13604 & 2,994 & 27 & ,006 \\
\hline Não & $\begin{array}{c}\text { 1a Coleta } \\
\text { ATTM-u (mg/g } \\
\text { de creatinina) - } \\
\text { 2a Coleta } \\
\text { ATTM-u (mg/g } \\
\text { de creatinina) }\end{array}$ & ,03500 & ,22072 & ,04030 & ,04742 & ,11742 & ,869 & 29 & ,392 \\
\hline
\end{tabular}

Legenda: $\mathrm{t}$ = teste $\mathrm{t}$ de student; $\mathrm{gl}=$ graus de liberdade; $\mathrm{sig}$ = significância. Fonte: Maiorquin CFR, 2015.

A Tabela 2, descritiva para idade, coleta de ATTM urinário e tempo de emprego mostra as médias dos resultados encontrados na primeira e segunda coleta de ATTM urinário, antes e após a intervenção com a utilização do equipamento de proteção individual respectivamente.

Tabela 2 - Descritiva para idade, coleta de ATTM-u e tempo no emprego.

\begin{tabular}{llllllll}
\hline Descritiva & Média & Mediana & $\begin{array}{l}\text { Desvio } \\
\text { Padrão }\end{array}$ & Min & Max & N & IC \\
\hline Idade & 28,2 & 27 & 7,4 & 18 & 54 & 58 & 1,9 \\
1 a Coleta ATTM-u & 0,22 & 0,17 & 0,21 & 0,01 & 1,13 & 58 & 0,05 \\
$2^{\text {a }}$ Coleta ATTM-u & 0,19 & 0,12 & 0,21 & 0,01 & 1,13 & 58 & 0,05 \\
Tempo no emprego & 1,9 & 0,8 & 2,8 & 0,5 & 15 & 58 & 0,7
\end{tabular}

Legenda: ATTM- $u=$ ácido trans, trans-mucônico urinário (mg/g de creatinina), Min= mínimo, Max= máximo, $\mathrm{N}=$ número, $\mathrm{IC}=$ intervalo de confiança a $95 \%$. Idade e tempo no emprego medido em anos.

Fonte: Maiorquin CFR, 2015.

\section{DISCUSSÃO}

O estudo permitiu conhecer a realidade da exposição dos trabalhadores mesmo em baixas concentrações de benzeno, após a análise pelo indicador biológico de exposição, o ATTM urinário, considerando a importância de seus efeitos sobre a saúde, de maneira que não há limite seguro para qualquer exposição ao carcinógeno (MENDES M, et al., 2017). Os indicadores de exposição ao benzeno (benzeno em sua forma original ou seus produtos de biotransformação) são achados no ar exalado, na urina e no sangue. 
A urina retida na bexiga por difusão tubular, tem sido considerada como uma amostra mais homogênea que o sangue e o ar expirado (SALGADO PET e PEZZAGNO G, 1991). Esta característica faz com que os metabólitos urinários sejam mais convenientes para a determinação da exposição ao benzeno em baixos níveis ambientais. Além da urina ter uma coleta mais fácil, ser um método não invasivo e ainda ser possível a coleta de grandes volumes para a amostra.

Conforme este estudo, e demais similares, nas exposições a substâncias com tempo de vida média relativamente curto, ou exposições com variações de intensidade durante a jornada de trabalho, se tem demonstrado que a urina coletada ao final da jornada de trabalho apresenta uma resposta mais eficaz às médias de exposição, do que o ar exalado e o sangue (HOET P, 1996).

Seguramente, a exposição ao benzeno nos ambientes de trabalho se acompanha do não cumprimento das normas de Segurança do Trabalho, da Legislação de Saúde eficaz ou ineficaz, má informação ou nenhuma informação inerente ao agente tóxico, supervisão inadequada, processos de trabalho e tecnologia obsoleta e falta ou mal-uso dos equipamentos de proteção individual.

O objetivo da pesquisa foi avaliar a eficácia do equipamento de proteção individual numa população exposta ao benzeno, considerando os níveis do ATTM urinário dos participantes que utilizaram ou não o mesmo. Houve uma relação significativa, sugerindo que o ATTM-u é um marcador biológico efetivo para avaliação da exposição ocupacional ao benzeno. A metodologia analítica utilizada para determinar o ATTM é simples, específica, sensível e prática.

A exposição ocupacional ao benzeno mostrada em nosso estudo, caracteriza a exposição a baixas concentrações do mesmo, uma vez que todos os valores estavam abaixo do valor de referência tecnológica (VRT) padrão.

Os níveis de ATTM-u achados no presente estudo coincidem com aqueles descritos na literatura. Segundo Paula FC, et al. (2003) este método de análise de ATTM-u é sensível para a determinação da concentração urinária deste metabólito do benzeno em concentrações urinárias a níveis abaixo de $0,04 \mathrm{mg} / \mathrm{g}$ de creatinina. Na pesquisa de Paula, as médias dos níveis de ATTM-u foram $0,19 \pm 0,04 \mathrm{mg} / \mathrm{g}$ de creatinina e nesta pesquisa a média foi $0,22 \mathrm{mg} / \mathrm{g}$ de creatinina (Tabela 2 ).

O equipamento de proteção individual se mostrou eficaz, considerando que houve uma diminuição estatisticamente significativa nos níveis de ATTM-u naqueles participantes que utilizaram o EPI em relação aos que não utilizaram.

Algumas limitações do método: a técnica utilizada avaliou a concentração do ATTM na urina somente ao final da jornada de trabalho. Eventos pontuais importantes durante o mesmo, podem ter sido desprezados. A pesquisa considera apenas algumas variáveis em um momento determinado, não sendo possível avaliar a influência destes no decorrer do tempo. A dieta com ácido sórbico e o tabagismo podem comprometer a especificidade do ATTM urinário como biomarcador de exposição (WEAVER VM, et al., 2000). Porém estas limitações não interferem de maneira alguma com a importância e necessidade da pesquisa.

Este é um estudo de correlação entre a exposição ao benzeno e os níveis de ATTM e de acordo com suas características representa o tipo de exposição ocupacional.

A partir da análise dos dados e informações apresentadas, observa-se que estamos diante de um problema de saúde pública complexo, onde as ações de promoção da saúde e proteção das populações sobre risco devem ser tomadas, estabelecendo prioridades e ações efetivas, observando padrões para o uso dos equipamentos de proteção individual, monitoramento dos limites de exposição ocupacional e estimativa da concentração do benzeno pelo tempo de jornada de trabalho (MENDES M, et al., 2017).

A exposição ocupacional ao agente cancerígeno tem um impacto importante na saúde pública. Estes níveis de exposição podem variar muito com os fatores físicos, ambientais e epidemiológicos, de acordo com a atividade que exerce cada trabalhador exposto (FALZONE L, et al., 2016). Existem variações de exposição mesmo entre indivíduos no mesmo local de trabalho, ressaltando a importância de aprofundar as pesquisas no campo dos cânceres ocupacionais, equipamentos de proteção individual e indicadores epidemiológicos.

Aqueles trabalhadores que operam diretamente na bomba de combustíveis estão mais próximos da fonte de vapor de benzeno, captando por inalação o mesmo. A função exercida pelo trabalhador tem um papel 
importante no efeito da exposição ao carcinógeno por hora trabalhada com relação aos riscos à saúde (CHAIKLIENG S, et al., 2019). Sugerimos comparação em estudos futuros, da exposição ocupacional ao benzeno, em trabalhadores da área da conveniência, lava-jato e parte administrativa em postos de combustíveis, com aqueles que trabalham junto a bomba de combustível em nova investigação, utilizando grupo controle e equipamento de proteção individual.

Este estudo recomenda um maior monitoramento da exposição ocupacional destes trabalhadores, controle de riscos, implementação de medidas de segurança, manutenção e prevenção de vazamentos de produtos químicos, adequação dos limites de exposição permitidos, enfatizando o uso contínuo e supervisão do uso de equipamento de proteção individual, bem como o incentivo e avanço nas pesquisas relacionadas a saúde do trabalhador (SALEM E, et al., 2018). A execução de políticas de saúde com programas e ações de conscientização, informação e prevenção, são fundamentais para a mudança do atual panorama.

\section{CONCLUSÃO}

Diante da impossibilidade de eliminar o benzeno do ambiente de trabalho, deve haver redução dos níveis de exposição ocupacional, difundir o conhecimento sobre os riscos e intensificar as medidas de prevenção. Esta pesquisa demonstra uma vez mais a importância da utilização de equipamentos de proteção individual para a saúde ocupacional e saúde pública e deve continuar sendo debatido e pesquisado, para garantir a proteção do trabalhador em seu ambiente de trabalho.

\section{AGRADECIMENTOS}

As análises bioquímicas do ATTM urinário foram contratadas pela autora e realizados pelo Farmacêutico/Bioquímico Dr. Antônio Francisco de Aguiar CRF-24 No 057, no Laboratório Bio Check-up, com apoio do Laboratório Hermes Pardini.

O Sindicato dos Trabalhadores de Comércio de Minérios e derivados de Petróleo do estado de RondôniaSTCMDP/RO, colaborou com os dados para cálculo amostral e na anuência e liberação da administração dos Postos de Gasolina para entrevistas com os participantes e realização da Pesquisa.

\section{REFERÊNCIAS}

1. ACGIH. American Conference of Governmental Industrial Hygienists. Threshold Limit Values (TLVs) for chemical substances and physical agents' biological exposure indices for 2013. Cincinnati, USA: ACGIH; 2013.

2. ACGIH. American Conference of Governmental Industrial Hygienists. TLV for benzene: CAS number: 71-43-2. Cincinnati, USA: American Conference of Governmental Industrial Hygienists; 2001.

3. ACGIH-American Conference of Governmental Industrial Hygienists. Threshold limit values for chemical substances and physical agents. Biological exposures indices. Cincinatti, 2006; 256p.

4. BRASIL, Ministério da Saúde. Fundação Nacional de Saúde. Centro Nacional de Epidemiologia. Guia de Vigilância Epidemiológica. Brasília, 1994; 117-123.

5. BRASIL. Ministério da Saúde. Doenças relacionadas ao trabalho: manual de procedimentos para o serviço de saúde. Brasília; 2001.Normas e Manuais Técnicos, 114p.

6. BRASIL. Ministério da Saúde. Risco Químico: atenção à saúde dos trabalhadores expostos ao benzeno. Brasília; 2006.

7. BRASIL. Ministério da Saúde. Secretaria de Atenção à Saúde. Departamento de Ações Programáticas Estratégicas. Câncer relacionado ao trabalho: leucemia mielóide aguda síndrome mielodisplásica decorrente da exposição ao benzeno. Brasília: Editora do Ministério da Saúde, 2006; 48p.

8. BRASIL. Ministério do Trabalho. Acordo e Legislação sobre Benzeno. Fundacentro, São Paulo, 1996.

9. Brasil. Ministério do Trabalho. Norma regulamentadora no 7 - Segurança e medicina do trabalho. Programa de Controle Médico de Saúde Ocupacional. São Paulo: Editora Atlas; 2010.

10. CERQUEIRA GS, et al. Exposição ocupacional de trabalhadores de postos de combustíveis do sertão Paraibano. Rev. Ciência e Saúde, 2010; 1(2): 310-331.

11. CHAIKLIENG S, et al. Avaliação de Riscos na Exposição ao Benzeno entre Trabalhadores em Postos de Gasolina. Int J Environ Res Saúde Pública, 2019; 16 (14): 2545.

12. CORRÊA MJM, LARENTIS AL. Exposição ao benzeno no trabalho e seus efeitos à saúde. Rev. bras. saúde ocup., 2017; 42(1): 14 p.

13. CORREA MJM, SANTANA VS. Exposição ocupacional ao benzeno no Brasil: estimativas baseadas em uma matriz de exposição ocupacional. Cad. Saúde Pública, 2016; 32(12): 1-11. 
14. COSTA MFB. Estudo da aplicabilidade do ácido trans,trans-mucônico urinário como indicador biológico de exposição ao benzeno. Tese (Doutorado em Saúde Pública) - Escola Nacional de Saúde Pública Sergio Arouca. Fundação Oswaldo Cruz, Rio de Janeiro, 2001; 126 p.

15. COSTA DF. Prevenção da Exposição de Benzeno no Brasil, SP. Tese (Doutorado em Ciências) - Universidade de São Paulo, São Paulo, 2009; 184 p.

16. FALZONE L. Exposição ocupacional a agentes cancerígenos: benzeno, pesticidas e fibras. Relatórios de medicina molecular, 2016; 14 (5), 4467-4474.

17. HOEPPNER MG. Normas Regulamentadoras relativas à Segurança e Medicina do Trabalho. 3a edição, 2008; 103p.

18. COTTICA D, GRIGNANI E. Evolution of technology and occupational exposures in petrochemical industry and in petroleum refining. G Ital Med Lav Ergon, 2013; 35(4): 236-243.

19. HOET P. Principios Generales. En: Salud ocupacional para todos: Monitoreo Biológico de Exposición a sustancias químicas en el lugar de trabajo. Ginebra: OMS, 1996; 1-19.

20. HUNTER FT. Chronic exposure to benzene.II.The clinical effects.J Ind Hyg Toxicol, 1939; 21:331-354.

21. IARC. International Agency for Research on Cancer. Benzene. Monograph Evaluation Carcinogenic Risk Chemical Human. 1989; 45:159-218.

22. MACEDO RB. Segurança, Saúde, Higiene e Medicina do Trabalho. Curitiba: IESDE, 2008; 128p.

23. MENDES M, et al. Normas ocupacionais do benzeno: uma abordagem sobre o risco e exposição nos postos de revenda de combustíveis. Rev. Bras. Saúde Ocup., 2017; 42(1): 1-19.

24. MENDES R. Patologia do Trabalho. 3.ed. Atheneu: Rio de Janeiro, 2013; 2000p.

25. MORIYAMA IN, et al. Prevenção da exposição ocupacional ao benzeno em trabalhadores de postos de revenda de combustíveis: a experiência do estado do Espírito Santo. Rev. bras. saúde ocup.,2017; 42(1): 4 p.

26. MORO AM, et al. Avaliação de biomarcadores de estresse imunológico, inflamatório e oxidativo em frentistas de postos de gasolina. BMC Pharmacol Toxicol, 2019; 20(1): 75 p.

27. OGA S et.al. Fundamentos de Toxicologia. Atheneu, 3aedição, 2008; 285p.

28. PAULA FCS, et al. Avaliação do ácido trans, trans-mucônico urinário como biomarcador de exposição ao benzeno. Rev. Saúde Pública, São Paulo, 2003; 37(6): 780-785.

29. PEZZANO G. Monitoraggio biologic delle populazioni exposte a benzene. Em minoia C, Apostoli P, Bartolucci GB(Eds). II Benzene: Tossicologia, ambienti di vida e de lavoro, 1995.

30. SALEM E, et al. Efeitos genotóxicos da exposição ocupacional ao benzeno em trabalhadores de postos de gasolina. Ind Health, 2018; 56 (2): 132-140.

31. SALGADO PET, PEZZAGNO G. Indicadores Biológicos de Exposição ao Benzeno. Revista Brasileira de Saúde Ocupacional, 1991; 19:25-31.

32. WEAVER VM, et al. Lack of specificity of trans, trans-muconic acid as a benzene biomarker after ingestion of sorbic acid-preserved foods. Cancer Epidemiol. Biomark. Prev., 2000; (9): 749-755.

33. WHO. Occupational Health for all Biological Monitoring of Chemical Exposure in the Workplace. World Health Organization. Geneva, 1996; 292p. 